\title{
Left atrial appendage size is a marker of atrial fibrillation recurrence after radiofrequency catheter ablation in patients with persistent atrial fibrillation
}

Judit Simon ${ }^{1}$, Mohammed El Mahdiui ${ }^{2}$, Jeff Smit $^{2}$, Lili Száraz ${ }^{3}$, Alexander van Rosendael ${ }^{2}$, Szilvia Herczeg ${ }^{4}$, Emese Zsarnóczay ${ }^{1}$, Anikó Nagy ${ }^{1}$, Márton Kolossváry ${ }^{3}$, Bálint Szilveszter $^{3}$, Nandor Szegedi ${ }^{3}$, Klaudia Vivien Nagy ${ }^{1}$, Tamás Tahin ${ }^{5}$, Laszlo Geller ${ }^{1}$, Rob van der Geest ${ }^{6}$, Jeroen Bax ${ }^{2}$, Pál Maurovich-Horvat ${ }^{3}$, and Bela Merkely ${ }^{1}$

${ }^{1}$ Semmelweis University

${ }^{2}$ Leiden University Medical Center

${ }^{3}$ Heart and Vascular Centre of Semmelweis University

${ }^{4}$ Heart and Vascular Center of Semmelweis University

${ }^{5}$ Semmelweis Egyetem

${ }^{6} \mathrm{LUMC}$

July 3, 2021

\begin{abstract}
Introduction Catheter ablation is an established therapy for rhythm control in patients with drug-refractory atrial fibrillation (AF), however, recurrence is frequent particularly in persistent AF. There are no consistently confirmed predictors of AF recurrence after catheter ablation. Therefore, we aimed to study whether LAA volume (LAAV) and function influence the longterm recurrence of AF after catheter ablation, depending on AF type. Methods AF patients who underwent point-by-point radiofrequency catheter ablation after cardiac computed tomography (CT) were included in this analysis. LAAV and LAA orifice area were measured by CT. Uni- and multivariable Cox proportional hazard regression models were performed to determine the predictors of AF recurrence. Results In total, 561 AF patients (61.9?10.2 years, $34.9 \%$ females) were included in the study. Recurrence of AF was detected in $40.8 \%$ of the cases (34.6\% in patients with paroxysmal and $53.5 \%$ in those with persistent AF) with a median recurrence-free time of 22.7 ?9.3-43.1? months. Patients with persistent AF had significantly higher iLAV, LAAV, LAA orifice area and lower LAA flow velocity, than those with paroxysmal AF. After adjustment left ventricular ejection fraction $(\mathrm{LVEF})<50 \%(\mathrm{HR}=2.17 ; 95 \% \mathrm{CI}=1.38-3.43 ; \mathrm{p}<0.001)$ and $\mathrm{LAAV}(\mathrm{HR}=1.06 ; 95 \% \mathrm{CI}=1.01-1.12 ; \mathrm{p}=0.029)$ were independently associated with AF recurrence in persistent AF, while no independent predictors could be identified in paroxysmal AF. Conclusion The current study demonstrates that beyond left ventricular systolic dysfunction, LAA enlargement is associated with higher rate of AF recurrence after catheter ablation in persistent AF, but not in patients with paroxysmal AF.
\end{abstract}

\section{Title}

Left atrial appendage size is a marker of atrial fibrillation recurrence after radiofrequency catheter ablation in patients with persistent atrial fibrillation

\section{Short title}

Predictors of atrial fibrillation recurrence

\section{Authors}


Judit Simon, $\mathrm{MD}^{1,2 *}$, Mohammed El Mahdiui, $\mathrm{MD}^{3 *}$, Jeff M. Smit, $\mathrm{MD}^{3}$, Lili Száraz ${ }^{1}$, Alexander R. van Rosendael, $\mathrm{MD}^{3}$, Szilvia Herczeg $\mathrm{MD}^{4}$, Emese Zsarnóczay ${ }^{1}$, Anikó Ilona Nagy, MD, $\mathrm{PhD}^{4}$, Márton Kolossváry, $\mathrm{MD}, \mathrm{PhD}^{1}$, Bálint Szilveszter, $\mathrm{MD}, \mathrm{PhD}^{1}$, Nándor Szegedi, $\mathrm{MD}, \mathrm{PhD}^{4}$, Klaudia Vivien Nagy, $\mathrm{MD}$, $\mathrm{PhD}^{4}$, Tamás Tahin, $\mathrm{MD}^{4}$, László Gellér, $\mathrm{MD}, \mathrm{PhD}^{4}$, Rob J. van der Geest, $\mathrm{PhD}^{5}$, Jeroen J. Bax MD, $\mathrm{PhD}^{3,6}$, Pál Maurovich-Horvat MD, PhD, MPH, DSc ${ }^{1,2 \#}$, Béla Merkely MD, PhD, DSc ${ }^{1 \#}$

${ }^{*}$ Contributed equally and shared first authors

\# Contributed equally and shared last authors

\section{Affiliations}

${ }^{1}$ MTA-SE Cardiovascular Imaging Research Group, Heart and Vascular Center, Semmelweis University, Budapest, Hungary

${ }^{2}$ Medical Imaging Centre, Semmelweis University, Budapest, Hungary

${ }^{3}$ Department of Cardiology, Leiden University Medical Center, Leiden, The Netherlands

${ }^{4}$ Heat and Vascular Center, Semmelweis University, Budapest, Hungary

${ }^{5}$ Division of Image Processing, Leiden University Medical Center, Leiden, The Netherlands

${ }^{6}$ Heart Center, Turku University Hospital and University of Turku, Turku, Finland

\section{E-mail addresses}

Judit Simon, MD:simon.judit@med.semmelweis-univ.hu

Mohammed El Mahdiui, MD:M.el_Mahdiui@lumc.nl

Jeff M. Smit, MD:J.M.Smit@lumc.nl

Lili Száraz: liller.szaraz97@gmail.com

Alexander R.van Rosendael, MD:a.r.van.rosendael@gmail.com

Szilvia Herczeg MD:szilvi.herczeg@gmail.com

Emese Zsarnóczay:emese.zsarnoczay@gmail.com

Anikó Ilona Nagy, MD, PhD:anychophora@gmail.com

Márton Kolossváry, MD, PhD:martonandko@gmail.com

Bálint Szilveszter, MD, PhD:szilveszter.balint@gmail.com

Nándor Szegedi, MD, PhD:nandorszegedi@gmail.com

Klaudia Vivien Nagy, MD, PhD:nagyklaudiavivien@gmail.com

Tamás Tahin, MD: ttahin@gmail.com

László Gellér, MD, PhD:laszlo.geller@gmail.com

Rob J. van der Geest, PhD:R.J.van_der_Geest@lumc.nl

Jeroen J. Bax MD, PhD:j.j.bax@lumc.nl

Pál Maurovich-Horvat MD, PhD, MPH, DSc:maurovich.horvat@gmail.com

Béla Merkely MD, PhD, DSc:merkely.bela@kardio.sote.hu

\section{Corresponding author}

Pál Maurovich-Horvat MD PhD MPH DSc 
ORCID: 0000-0003-0885-736X

E-mail address:maurovich.horvat@gmail.com

Telephone: $+36 / 1-210-0300 / 53312$

Fax: $+36 / 1-459-1500 / 53171$

\section{Conflict of interest}

The authors have no conflicts to disclose.

\section{Word count}

4998

\section{Acknowledgements}

Project no. NVKP_16-1-2016-0017 ('National Heart Program') has been implemented with the support provided from the National Research, Development and Innovation Fund of Hungary, financed under the NVKP_16 funding scheme. The research was financed by the Thematic Excellence Programme (2020-4.1.1.-TKP2020) of the Ministry for Innovation and Technology in Hungary, within the framework of the Therapeutic Development and Bioimaging thematic programmes of the Semmelweis University.

\section{Abstract \\ Introduction}

Catheter ablation is an established therapy for rhythm control in patients with drug-refractory atrial fibrillation (AF), however, recurrence is frequent particularly in persistent AF. There are no consistently confirmed predictors of AF recurrence after catheter ablation. Therefore, we aimed to study whether LAA volume $(\mathrm{LAAV})$ and function influence the long-term recurrence of AF after catheter ablation, depending on AF type.

\section{Methods}

AF patients who underwent point-by-point radiofrequency catheter ablation after cardiac computed tomography $(\mathrm{CT})$ were included in this analysis. LAAV and LAA orifice area were measured by CT. Uni- and multivariable Cox proportional hazard regression models were performed to determine the predictors of AF recurrence.

\section{Results}

In total, $561 \mathrm{AF}$ patients (61.9 \pm 10.2 years, $34.9 \%$ females) were included in the study. Recurrence of $\mathrm{AF}$ was detected in $40.8 \%$ of the cases (34.6\% in patients with paroxysmal and $53.5 \%$ in those with persistent AF) with a median recurrence-free time of 22.7 [9.3-43.1] months. Patients with persistent AF had significantly higher iLAV, LAAV, LAA orifice area and lower LAA flow velocity, than those with paroxysmal AF. After adjustment left ventricular ejection fraction $(\mathrm{LVEF})<50 \%(\mathrm{HR}=2.17 ; 95 \% \mathrm{CI}=1.38-3.43 ; \mathrm{p}<0.001)$ and LAAV $(\mathrm{HR}=1.06 ; 95 \% \mathrm{CI}=1.01-1.12 ; \mathrm{p}=0.029)$ were independently associated with AF recurrence in persistent AF, while no independent predictors could be identified in paroxysmal AF.

\section{Conclusion}

The current study demonstrates that beyond left ventricular systolic dysfunction, LAA enlargement is associated with higher rate of $\mathrm{AF}$ recurrence after catheter ablation in persistent $\mathrm{AF}$, but not in patients with paroxysmal AF.

\section{Keywords}

Atrial fibrillation; echocardiography, transesophageal; echocardiography, transthoracal; heart atria; recurrence; tomography, X-Ray 


\section{Abbreviations}

AAD: anti-arrhythmic drug

AF: atrial fibrillation

BMI: body mass index

CAD: coronary artery disease

eGFR: estimated glomerular filtration rate

iLAV: body surface area-indexed left atrial volume

LA: left atrium

LAA: left atrial appendage

LAAV: left atrial volume

LVEF: left ventricular ejection fraction

PVI: pulmonary vein isolation

TIA: transient ischemic attack

\section{Introduction}

Atrial fibrillation (AF) is the most common sustained cardiac arrhythmia. ${ }^{1}$ It can lead to several health problems, such as heart failure, embolic events, and impaired quality of life. Moreover, it is associated with higher mortality rate. ${ }^{2-4}$ In case of drug-resistant symptomatic AF, catheter ablation proved to be an effective solution for rhythm control. ${ }^{5}$ However, depending on the ablation strategy and the type of $\mathrm{AF}$, success rates of catheter ablation after 1 year vary considerably from $60 \%$ to $90 \% \cdot{ }^{5-7}$ Appropriate patient selection for catheter ablation is essential as neither AF recurrence nor procedural complication rates are negligible. ${ }^{8}$ However, there are no consistently confirmed predictors of AF recurrence following catheter ablation in the literature. ${ }^{9-18}$ It has been suggested that left atrial appendage (LAA) volume and function may be associated with the recurrence of $\mathrm{AF} /$ tachycardia in patients undergoing repeated ablation, the exact role of the LAA in the prediction of AF recurrence has not yet been resolved. ${ }^{19}$ The anatomy, including LAA volume, morphology and LAA orifice area can be accurately described using cardiac computed tomography (CT), and LAA function can be assessed by measuring LAA flow velocity using transesophageal echocardiography (TEE).

We aimed to study the role of LAA volume (LAAV), and function in the success of catheter ablation by type of AF.

\section{Methods}

\section{Study population}

In our multimodality retrospective study, we included consecutive patients with symptomatic AF who underwent initial point-by-point radiofrequency catheter ablation at the Heart and Vascular Center of Semmelweis University, Budapest, Hungary between January 2014 and December 2017. All patients underwent preprocedural cardiac CT for the assessment of left atrial (LA) anatomy. LAA function was assessed with LAA flow velocity measured by TEE. Left ventricular ejection fraction (LVEF) was measured by transthoracic echocardiography. Exclusion criteria were age under 18 years, non-diagnostic CT image quality, history of prior pulmonary vein isolation or heart surgery, and missing echocardiography data on LAA flow velocity. Due to the retrospective analysis of clinically acquired data, the institutional review board waived the need for written patient informed consent. The study was performed according to the Declaration of Helsinki and Institutional Guidelines.

Cardiac CT imaging 
CT examinations were performed with a 256-slice scanner (Brilliance iCT 256, Philips Healthcare, Best, The Netherlands) with prospective ECG-triggered axial acquisition mode. 100-120 kV with 200-300 mAs tube current was used depending on patient anthropometrics. Image acquisition was performed with $128 \mathrm{x} 0.625$ $\mathrm{mm}$ detector collimation, and $270 \mathrm{msec}$ gantry rotation time. For heart rate control 50-100 mg metoprolol was given orally and 5-20 mg intravenously, if necessary. In total $85-95 \mathrm{ml}$ contrast material (Iomeron 400, Bracco Ltd, Milan, Italy) was injected with a flow rate of $4.5-5.5 \mathrm{ml} / \mathrm{sec}$ via antecubital vein access using a four-phasic injection protocol. ${ }^{20}$ Bolus tracking in the LA was used to obtain proper scan timing. All patients received $0.8 \mathrm{mg}$ sublingual nitroglycerin between the native CT and CTA examinations. CT data sets were reconstructed with $0.8 \mathrm{~mm}$ slice thickness and $0.4 \mathrm{~mm}$ increments. The LA and LAA volumes were measured using a semiautomatic software tool (EP Planning, Philips IntelliSpace Portal, Philips Healthcare, Best, The Netherlands) and if needed the borders of LA and LAA, the orifices of the pulmonary veins and the level of the mitral valve were manually adjusted.

\section{LAA flow velocity measurement}

Maximum 24h before ablation, all patients underwent TEE examination to exclude the presence of LAA thrombus. iE33 and Epiq 7C (Philips Medical System, Andover, MA) systems equipped with S5-1 phased array and X7-2t matrix TEE transducers were used. TEE was performed during conscious sedation. The LAA was imaged from $0^{\circ}, 45^{\circ}, 90^{\circ}$ and $135^{\circ}$ views to detect spontaneous echo contrast, sludge or thrombus. Subsequently, a sample volume was placed at the middle portion of the LAA and the peak velocity of the outflow of the LAA was measured.

\section{Catheter ablation procedure}

The indications for AF ablation procedures were in accordance with the current guidelines. ${ }^{1,}{ }^{21}$ Paroxysmal AF was defined as self-terminating AF, in most cases within 48 hours. Some AF paroxysms continued up to 7 days. ${ }^{21}$ Persistent AF was defined as AF that lasts longer than 7 days. ${ }^{21}$ Conscious sedation was carried out in all cases with intravenous fentanyl, midazolam, and propofol. Basic vital parameters of the patients were monitored in all cases with non-invasive blood pressure measurements every 10 minutes and continuous pulse oximetry. Femoral venous access was used for all procedures. Transseptal puncture was performed routinely with fluoroscopy guidance and pressure monitoring, while intracardiac echocardiography was also utilized in difficult cases. All ablations were performed with the support of an electroanatomical mapping system (either CARTO, Biosense Webster, Inc., Diamond Bar, CA, USA; or ENSITE, St. Jude Medical, Inc., MN, USA), and the LA fast anatomical map was fused with the cardiac CT images to guide ablation (temperature-controlled mode, $43 \mathrm{degC}, 25-35 \mathrm{~W}$, irrigated $4 \mathrm{~mm}$ tip catheter) in the majority of patients. Pulmonary vein isolation was performed in each patient. Moreover, in patients with long-standing persistent $\mathrm{AF}$, additional ablation lines were drawn at the discretion of the operating physician. All patients without complications were discharged the day after the procedure.

Follow-up and definition of AF recurrence

After discharge, outpatient clinical follow-up visits were scheduled at 3, 6, 12 months after the procedure and at least once yearly thereafter. The follow-up visits included clinical assessment of the patient and 24-hour Holter ECG monitoring. Follow-up data were registered in the structured reporting platform (Axis, Neumann Medical Ltd, Budapest, Hungary). Recurrence of AF was defined as the occurrence of atrial tachyarrhythmia that lasted for more than 30 sec. ${ }^{1,21}$ AF recurrences during the first 90 days after catheter ablation were not included in order to exclude AF during this vulnerable ,,blanking period", which might be only a temporary phenomenon due to the inflammation, maturation and healing of the ablated lesions. ${ }^{22,} 23$

Statistical analysis

Categorical variables are expressed as frequencies (percentages) and continuous values are expressed as mean+-standard deviation (SD). Normality of continuous parameters was tested with Shapiro-Wilk test. Tests for significance were conducted using Mann-Whitney-Wilcoxon or Kruskal-Wallis tests for continuous variables and Pearson's chi-square or Fisher exact tests (in case of 5 or less observations) for categorical 
variables. The event-free survival rate was estimated using Kaplan-Meier method and log-rank test was applied for the comparisons between the various groups. Cumulative event rates were calculated with event or censoring times measured from the date of ablation. For patients who did not experience AF recurrence, their time-to-event measure was censored at the last follow-up visit date.

To identify parameters associated with AF recurrence after catheter ablation, uni- and multivariate Cox proportional hazard regression model was executed. In the multivariate model, adjustment was made for age $>65$ years, persistent AF, impaired eGFR $\left(<60 \mathrm{ml} / \mathrm{min} / 1.73 \mathrm{~m}^{2}\right)$, body surface area-indexed LA volume (iLAV) measured by CT, LVEF $<50 \%$ ), sex, obesity (defined as body mass index[?]30kg $/ \mathrm{m}^{2}$ ), hypertension, dyslipidemia, diabetes (Type I and II), prior stroke/transient ischemic attack (TIA), obstructive coronary artery disease (CAD), thyroid gland diseases (hypo- and hyperthyroidism), unsuccessful preablational antiarrhythmic drug (AAD) therapy (including sotalol, propafenone and amiodarone therapies), and LAAV. Thirty patients were randomly selected for interobserver agreement and analyzed using intraclass correlation coefficient (ICC). Relative risks were expressed as hazard ratios (HRs) with associated 95\% confidence intervals (CIs). Two-tailed p values smaller than 0.05 were considered significant. All statistical analyses were performed in $\mathrm{R}$ environment (version 3.6.1). Cox proportional hazard regression analysis was done using the 'survival' package (version 3.1-8). Kaplan-Meier curve and log-rank test were performed using the 'survminer' (version 0.4.6).

\section{Results}

Patient characteristics

A total of 561 patients were included in the current analysis. Mean age was $61.9+-10.2$ years and $34.9 \%$ of the patients were female. Recurrence of AF was reported in $40.8 \%$ of the patients (34.6\% in patients with paroxysmal and $53.5 \%$ in those with persistent AF). Median recurrence-free time was 22.7 [9.3-43.1] months (21.8 [9.4-43.2] months in paroxysmal and 23.6 [9.0-42.6] months in persistent AF. An excellent interobserver agreement was obtained for both the $\mathrm{iLAV}(\mathrm{ICC}=0.99)$, and LAAV $(\mathrm{ICC}=0.90)$ measurements. The proportion of individuals aged $>65$ years $(40.7 \%$ vs $49.3 \%$; $\mathrm{p}=0.046)$, female gender $(30 \%$ vs $41.9 \%$; $\mathrm{p}=0.005)$, persistent $\mathrm{AF}(25.9 \%$ vs $43.2 \%$; p $<0.001)$, and $\mathrm{LVEF}<50 \%$ (6.9\% vs $21.0 \%$; $\mathrm{p}<0.001)$ were significantly higher in patients with AF recurrence. Moreover, patients with AF recurrence had significantly higher iLAV $\left(54.4+-19.3 \mathrm{ml} / \mathrm{m}^{2}\right.$ vs $\left.61.8+-23.9 \mathrm{ml} / \mathrm{m}^{2} ; \mathrm{p}<0.001\right)$, LAAV $(7.6+-3.2 \mathrm{ml}$ vs $8.8+-5.2 \mathrm{ml} ; \mathrm{p}=0.002)$ and LAA orifice area $\left(387.6+-140.5 \mathrm{~mm}^{2}\right.$ vs $454.4+-167.7 \mathrm{~mm}^{2}$; $\left.\mathrm{p}<0.001\right)$. Anthropometric data, cardiovascular comorbidities, AAD therapy and imaging parameters are summarized in Table 1. Medications and procedural times are reported in Supplementary table 1.

We also examined the differences of the clinical and imaging parameters between patients with paroxysmal and persistent AF. Those patients with persistent AF had significantly higher proportion of age $>65$ years (41.0\% vs $50.8 \%$; $\mathrm{p}=0.030)$, hypertension $(67 \%$ vs $85.9 \%$; $<<0.001)$ and LVEF $<50 \%$ (6.6\% vs $24.9 \%$; $\mathrm{p}<0.001)$. Regarding the CT parameters, we measured significantly higher iLAV $\left(51.0+-15.9 \mathrm{ml} / \mathrm{m}^{2}\right.$ vs $\left.70.4+-25.6 \mathrm{ml} / \mathrm{m}^{2} ; \mathrm{p}<0.001\right)$, LAAV $(7.4+-3.0 \mathrm{ml}$ vs $9.5+-5.6 \mathrm{ml} ; \mathrm{p}=0.002)$, LAA orifice area $(385.2+-132.8$ $\mathrm{mm}^{2}$ vs $\left.475.2+-179.7 \mathrm{~mm}^{2} ; \mathrm{p}<0.001\right)$ and lower LAA flow velocity $(35.3+-13.4 \mathrm{~cm} / \mathrm{sec}$ vs $31.7+-12.0 \mathrm{~cm} / \mathrm{sec}$; $\mathrm{p}<0.001)$. Detailed data on the clinical and imaging parameters by AF type can be seen in Table 2 and Figure 1.

\section{Predictors of AF recurrence}

Significantly higher iLAV and LAAV values were measured in patients with persistent AF recurrences, and larger LAA orifice area values were measured both in paroxysmal and persistent recurrences, as reported in Figure 1. To explore the associations between the various examined parameters and AF recurrence, Cox proportional hazards regression analyses were performed, as stratified by AF type. In the univariate analysis, female sex $(\mathrm{HR}=1.43 ; 95 \% \mathrm{CI}=1.01-2.02 ; \mathrm{p}=0.043)$ was significantly associated with $\mathrm{AF}$ recurrence in patients with paroxysmal AF, while in those with persistent AF LVEF $<50 \%(\mathrm{HR}=2.07 ; 95 \% \mathrm{CI}=1.36-3.14$; $\mathrm{p}<0.001)$, iLAV $(\mathrm{HR}=1.01 ; 95 \% \mathrm{CI}=1.00-1.02 ; \mathrm{p}=0.027)$, LAAV $(\mathrm{HR}=1.07 ; 95 \% \mathrm{CI}=1.03-1.10 ; \mathrm{p}<0.001)$ and LAA orifice area $\left(\mathrm{HR}=1.02 ; 95 \% \mathrm{CI}=1.00-1.03\right.$ per $\left.10 \mathrm{~mm}^{2} ; \mathrm{p}=0.005\right)$ showed an association with $\mathrm{AF}$ 
recurrence.

After adjustment LVEF $<50 \%(\mathrm{HR}=2.17 ; 95 \% \mathrm{CI}=1.38-3.43 ; \mathrm{p}<0.001)$ and LAAV $(\mathrm{HR}=1.06 ; 95 \% \mathrm{CI}=1.01-$ $1.12 ; \mathrm{p}=0.029)$ remained a significant predictor of $\mathrm{AF}$ recurrence in patients with persistent AF, while in paroxysmal AF no independent predictors could be identified in the multivariate analysis. Detailed results of the uni- and multivariate Cox regression analyses are reported in Table 3. Kaplan-Meier curves of AF recurrence-free survival in persistent AF stratified by LVEF and LAAV can be seen in Figure 2.

\section{Discussion}

We demonstrated that beyond impaired LVEF, a larger LAAV is an independent predictor of AF recurrence after catheter ablation in patients with persistent AF. Interestingly, this association was not present in patients with paroxysmal AF.

$\mathrm{AF}$ is a complex disease with incompletely understood mechanisms. Although significant progress has been made in the last two decades, the efficacy of ablation therapy remains suboptimal, particularly in persistent AF. One-year success rate of catheter ablation varies between 60 and 90\%. ${ }^{5-7}$ Previous studies have shown that the majority of AF recurrence occurred in the first two years after catheter ablation. ${ }^{24}$ So far, persistent AF, LA enlargement, hypertension, diabetes mellitus, aging, obesity, heart failure, chronic renal insufficiency and preprocedural amiodarone failure have been reported as independent predictors of AF recurrence. $^{1,21,24-27}$ However, the data are controversial and the conclusions of previous studies are inconsistent. Several studies aimed to investigate the role of different scoring systems in the prediction of rhythm outcomes after AF ablation. While the HATCH score was found to have no value in the prediction of AF recurrence after catheter ablation, $\mathrm{R}_{2} \mathrm{CHADS} \mathrm{S}_{2}$ and $\mathrm{CHA}_{2} \mathrm{DS}_{2}$-VASc scores were associated with rhythm outcomes. ${ }^{28,29}$ Since APPLE score proved to be superior to the $\mathrm{CHA}_{2} \mathrm{DS}_{2}$-VASc score for the prediction of rhythm outcome after catheter ablation, we incorporated its factors into our multivariable models. ${ }^{30}$ Due to inconsistent definition of recurrence, estimation of the AF ablation success is challenging. 1,21 Current guidelines of the European Society of Cardiology define AF recurrence as the occurrence of atrial tachyarrhythmia that last for more than 30 sec. $^{1,}{ }^{21}$ In the current study, we have also applied this definition. Moreover, previous studies have reported a wide range of recurrent AF duration time following various ablation strategies. In our study, we included AF patients who underwent point-by-point catheter ablation procedure after 2014 in order to provide more useful information to the current clinical practice. Moreover, since efficacy of radiofrequency catheter ablation varies greatly between paroxysmal and persistent AF, we analyzed the outcomes separately by type of AF. Since radiofrequency catheter ablation procedure became more widely performed, clinical studies regarding the long-term effectiveness are important, especially in patients with persistent AF. In our study population the recurrence rate after point-by-point catheter ablation was $53.5 \%$ in persistent AF and 34.6 in paroxysmal AF after a single procedure. These findings suggest that catheter ablation in patients with persistent AF should be chosen very cautiously due to the low success rate and non-negligible complication rate. In the present study, left ventricular systolic dysfunction and higher LAAV were identified as significant predictors of AF recurrence in patients with persistent AF who underwent point-by-point catheter ablation.

Di Biase et al have reported that LAA appears to be responsible for recurrence of AF/tachycardia in at least $27 \%$ of patients undergoing repeated ablation, especially in persistent AF cases. ${ }^{19}$ Moreover, electric isolation of the LAA was associated with an improved AF-free/recurrence-free survival. ${ }^{31}$ Despite the increasing evidence of the role of LAA in triggering atrial arrhythmias, the literature is scarce regarding the contributing factors. Previous smaller studies including both paroxysmal and persistent AF patients undergoing catheter

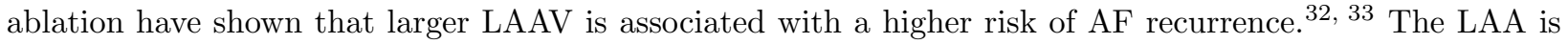
known to be more compliant than the LA, and therefore may play an important role in the modulation of LA pressure and LAAV measurement could be a reliable tool in determining the structural and functional conditions of LA from the early stage of AF. ${ }^{34}$ In line with these findings, our results also suggest that LAAV may be a surrogate of increased LAA arrhythmogenicity. LAA has a complex anatomy and LAA enlargement might result in longer activation pathways and development of re-entry through interstitial fibrosis. ${ }^{35}$ 
The current study has several limitations. First, it was a single-center, retrospective study, therefore the results need further validation in prospective, multi-center studies. Second, even if patients were closely followed-up with regular clinical visits, ECG and Holter monitoring, asymptomatic AF episodes might have happened between two visits. Moreover, ablation strategy was not uniform in all subjects, as additional ablations were permitted beyond PVI if operator decided to draw additional lines.

\section{Conclusion}

Despite advances in catheter ablation techniques, AF recurrence rate is relatively high. The current study demonstrates that beyond left ventricular systolic dysfunction, LAA enlargement is an independent predictor of AF recurrence after catheter ablation in persistent AF. Our results suggest that preprocedural assessment of LVEF and LAAV might contribute to optimal patient selection and aid to improve long-term results of ablation procedures in patients with persistent $\mathrm{AF}$.

\section{Acknowledgements}

Project no. NVKP_16-1-2016-0017 ('National Heart Program') has been implemented with the support provided from the National Research, Development and Innovation Fund of Hungary, financed under the NVKP_16 funding scheme. The research was financed by the Thematic Excellence Programme (2020-4.1.1.TKP2020) of the Ministry for Innovation and Technology in Hungary, within the framework of the Therapeutic Development and Bioimaging thematic programmes of the Semmelweis University.

\section{References}

1. Calkins H, Hindricks G, Cappato R, et al. 2017 HRS/EHRA/ECAS/APHRS/SOLAECE expert consensus statement on catheter and surgical ablation of atrial fibrillation: Executive summary. J Arrhythm Oct 2017;33:369-409.

2. Dorian P, Jung W, Newman D, et al. The impairment of health-related quality of life in patients with intermittent atrial fibrillation: implications for the assessment of investigational therapy. J Am Coll Cardiol Oct 2000;36:1303-1309.

3. Wang TJ, Larson MG, Levy D, et al. Temporal relations of atrial fibrillation and congestive heart failure and their joint influence on mortality: the Framingham Heart Study. Circulation Jun 17 2003;107:2920-2925.

4. Wolf PA, Abbott RD, Kannel WB. Atrial fibrillation as an independent risk factor for stroke: the Framingham Study. Stroke Aug 1991;22:983-988.

5. Oral H, Pappone C, Chugh A, et al. Circumferential pulmonary-vein ablation for chronic atrial fibrillation. N Engl J Med Mar 2 2006;354:934-941.

6. Cappato R, Calkins H, Chen SA, et al. Worldwide survey on the methods, efficacy, and safety of catheter ablation for human atrial fibrillation. Circulation Mar 8 2005;111:1100-1105.

7. Wazni OM, Marrouche NF, Martin DO, et al. Radiofrequency ablation vs antiarrhythmic drugs as first-line treatment of symptomatic atrial fibrillation: a randomized trial. JAMA Jun 1 2005;293:2634-2640.

8. Szegedi N, Szeplaki G, Herczeg S, et al. Repeat procedure is a new independent predictor of complications of atrial fibrillation ablation. Europace May 1 2019;21:732-737.

9. Bhargava M, Di Biase L, Mohanty $\mathrm{P}$, et al. Impact of type of atrial fibrillation and repeat catheter ablation on long-term freedom from atrial fibrillation: results from a multicenter study. Heart Rhythm Oct 2009;6:1403-1412.

10. Gerstenfeld EP, Sauer W, Callans DJ, et al. Predictors of success after selective pulmonary vein isolation of arrhythmogenic pulmonary veins for treatment of atrial fibrillation. Heart Rhythm Feb 2006;3:165-170.

11. Helms AS, West JJ, Patel A, et al. Relation of left atrial volume from three-dimensional computed tomography to atrial fibrillation recurrence following ablation. Am J Cardiol Apr 1 2009;103:989-993. 
12. Li C, Ding X, Zhang J, Zhou C, Chen Y, Rao L. Does the E/e' index predict the maintenance of sinus rhythm after catheter ablation of atrial fibrillation? Echocardiography Jul 2010;27:630-636.

13. Lim TW, Jassal IS, Ross DL, Thomas SP. Medium-term efficacy of segmental ostial pulmonary vein isolation for the treatment of permanent and persistent atrial fibrillation. Pacing Clin Electrophysiol Apr 2006;29:374-379.

14. Park SM, Kim YH, Choi JI, Pak HN, Kim YH, Shim WJ. Left atrial electromechanical conduction time can predict six-month maintenance of sinus rhythm after electrical cardioversion in persistent atrial fibrillation by Doppler tissue echocardiography. J Am Soc Echocardiogr Mar 2010;23:309-314.

15. Tilz RR, Rillig A, Thum AM, et al. Catheter ablation of long-standing persistent atrial fibrillation: 5-year outcomes of the Hamburg Sequential Ablation Strategy. J Am Coll Cardiol Nov 6 2012;60:1921-1929.

16. Vasamreddy CR, Lickfett L, Jayam VK, et al. Predictors of recurrence following catheter ablation of atrial fibrillation using an irrigated-tip ablation catheter. J Cardiovasc Electrophysiol Jun 2004;15:692-697.

17. Yasuda T, Kumagai K, Ogawa M, et al. Predictors of successful catheter ablation for atrial fibrillation using the pulmonary vein isolation technique. J Cardiol Aug 2004;44:53-58.

18. Berruezo A, Tamborero D, Mont L, et al. Pre-procedural predictors of atrial fibrillation recurrence after circumferential pulmonary vein ablation. Eur Heart J Apr 2007;28:836-841.

19. Di Biase L, Burkhardt JD, Mohanty P, et al. Left atrial appendage: an underrecognized trigger site of atrial fibrillation. Circulation Jul 13 2010;122:109-118.

20. Karady J, Panajotu A, Kolossvary M, et al. The effect of four-phasic versus three-phasic contrast media injection protocols on extravasation rate in coronary CT angiography: a randomized controlled trial. Eur Radiol Nov 2017;27:4538-4543.

21. Kirchhof P, Benussi S, Kotecha D, et al. 2016 ESC Guidelines for the management of atrial fibrillation developed in collaboration with EACTS. Europace Nov 2016;18:1609-1678.

22. Deftereos S, Giannopoulos G, Kossyvakis C, et al. Colchicine for prevention of early atrial fibrillation recurrence after pulmonary vein isolation: a randomized controlled study. J Am Coll Cardiol Oct 30 2012;60:1790-1796.

23. European Heart Rhythm A, European Cardiac Arrhythmia S, American College of C, et al. HRS/EHRA/ECAS expert Consensus Statement on catheter and surgical ablation of atrial fibrillation: recommendations for personnel, policy, procedures and follow-up. A report of the Heart Rhythm Society (HRS) Task Force on catheter and surgical ablation of atrial fibrillation. Heart Rhythm Jun 2007;4:816-861.

24. Mujovic NM, Marinkovic MM, Potpara TS, Geller L. Catheter ablation of lone atrial fibrillation. Curr Pharm Des 2015;21:591-612.

25. Hussein AA, Saliba WI, Martin DO, et al. Natural history and long-term outcomes of ablated atrial fibrillation. Circ Arrhythm Electrophysiol Jun 2011;4:271-278.

26. Mohanty S, Mohanty P, Di Biase L, et al. Impact of metabolic syndrome on procedural outcomes in patients with atrial fibrillation undergoing catheter ablation. J Am Coll Cardiol Apr 3 2012;59:1295-1301.

27. Shah AN, Mittal S, Sichrovsky TC, et al. Long-term outcome following successful pulmonary vein isolation: pattern and prediction of very late recurrence. J Cardiovasc Electrophysiol Jul 2008;19:661-667.

28. Kornej J, Hindricks G, Kosiuk J, et al. Comparison of CHADS2, R2CHADS2, and CHA2DS2-VASc scores for the prediction of rhythm outcomes after catheter ablation of atrial fibrillation: the Leipzig Heart Center AF Ablation Registry. Circ Arrhythm Electrophysiol Apr 2014;7:281-287.

29. Tang RB, Dong JZ, Long DY, et al. Efficacy of catheter ablation of atrial fibrillation beyond HATCH score. Chin Med J (Engl) Oct 2012;125:3425-3429. 
30. Kornej J, Hindricks G, Shoemaker MB, et al. The APPLE score: a novel and simple score for the prediction of rhythm outcomes after catheter ablation of atrial fibrillation. Clin Res Cardiol Oct 2015;104:871-876.

31. Di Biase L, Burkhardt JD, Mohanty P, et al. Left Atrial Appendage Isolation in Patients With Longstanding Persistent AF Undergoing Catheter Ablation: BELIEF Trial. J Am Coll Cardiol Nov 1 2016;68:1929-1940.

32. Zheng GA, Lin CY, Weng L, Chen JD. [Left atrial appendage volume is a valuable predictor of atrial fibrillation recurrence after radiofrequency catheter ablation]. Zhonghua Xin Xue Guan Bing Za Zhi Nov 24 2017;45:924-929.

33. Pinto Teixeira P, Martins Oliveira M, Ramos R, et al. Left atrial appendage volume as a new predictor of atrial fibrillation recurrence after catheter ablation. J Interv Card Electrophysiol Aug 2017;49:165-171.

34. Tabata $\mathrm{T}$, Oki $\mathrm{T}$, Yamada $\mathrm{H}$, et al. Role of left atrial appendage in left atrial reservoir function as evaluated by left atrial appendage clamping during cardiac surgery. Am J Cardiol Feb 1 1998;81:327-332.

35. Krul SP, Berger WR, Smit NW, et al. Atrial fibrosis and conduction slowing in the left atrial appendage of patients undergoing thoracoscopic surgical pulmonary vein isolation for atrial fibrillation. Circ Arrhythm Electrophysiol Apr 2015;8:288-295.

\section{Figures}

Figure 1. Comparison of LA and LAA parameters between patients with and without AF recurrence stratified by AF type
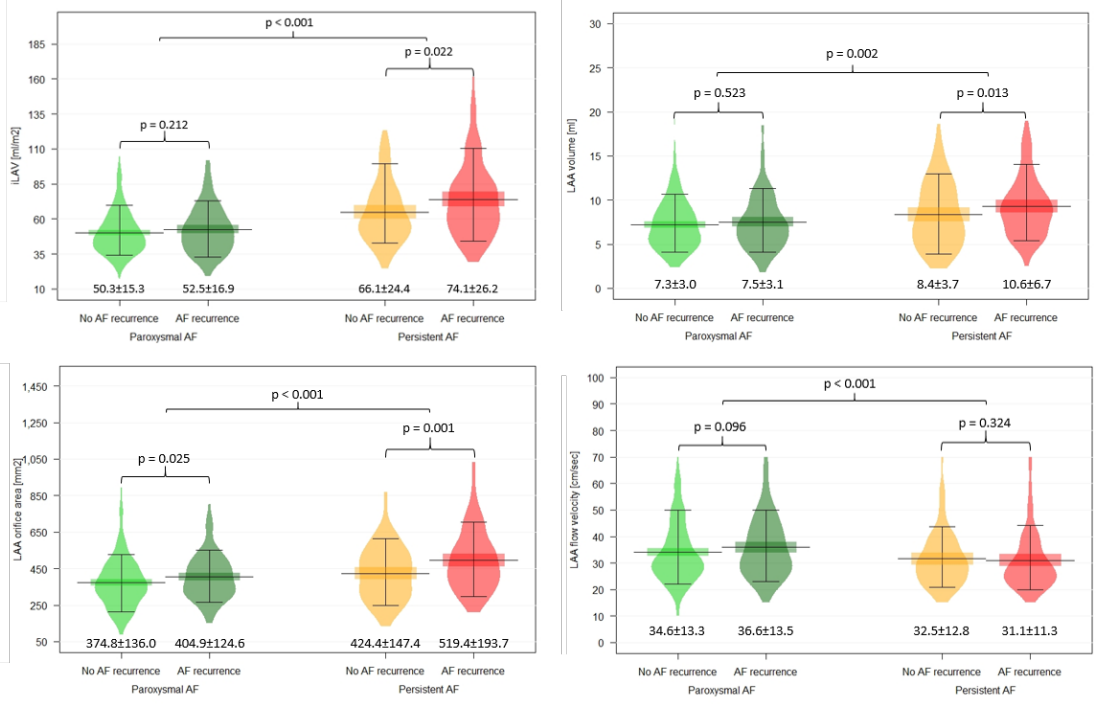

Abbrevations: $\mathrm{AF}=$ atrial fibrillation; iLAV = body surface area-indexed left atrial volume; LAA = left atrial appendage.

Figure 2. AF recurrence-free survival according to LVEF and LAAV in patients with persistent AF 


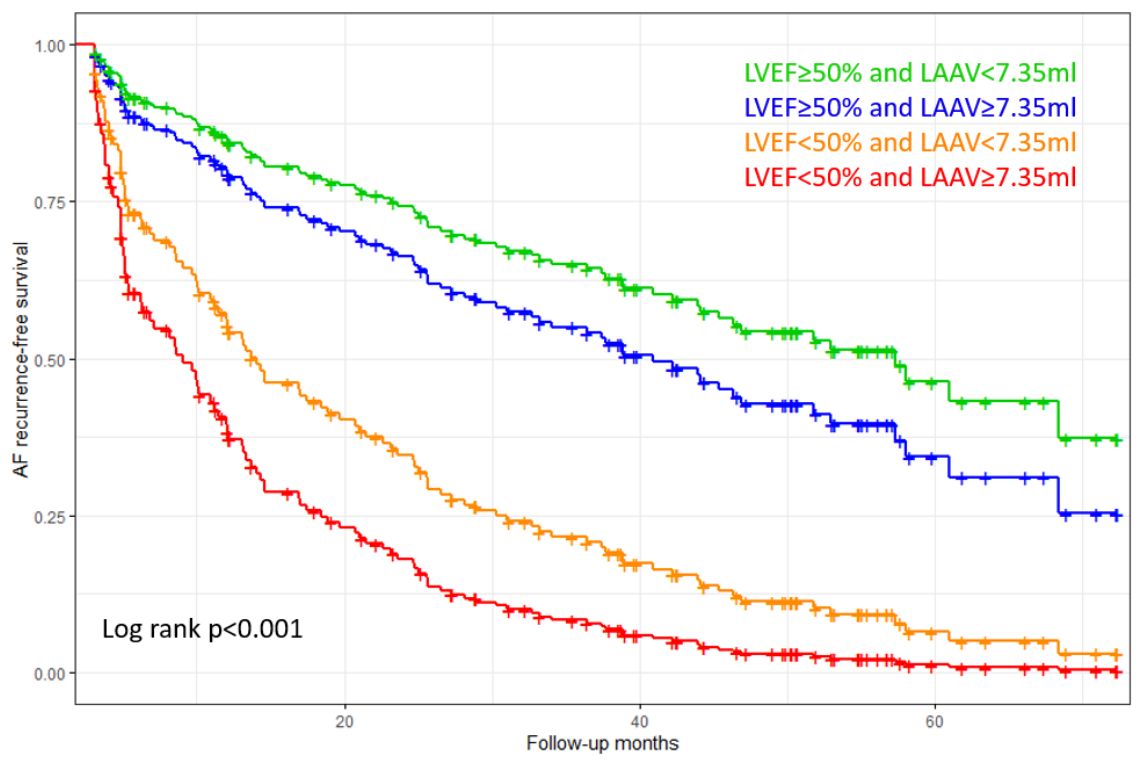

Abbreviations: $\mathrm{AF}=$ atrial fibrillation; $\mathrm{LAAV}=$ left atrial appendage volume; $\mathrm{LVEF}=$ left ventricular ejection fraction.

\section{Tables}

Table 1. Patient characteristics

\begin{tabular}{lll}
\hline & All patients $(\mathrm{n}=561)$ & No AF recurrence $(\mathrm{n}=332)$ \\
\hline Anthropometric data and comorbidities & Anthropometric data and comorbidities & Anthropometric data a \\
Age $>65$ years, $\mathrm{n}(\%)$ & $248(44.2)$ & $135(40.7)$ \\
Female, $\mathrm{n}(\%)$ & $561(34.9)$ & $100(30.1)$ \\
Persistent AF, n (\%) & $185(33.0)$ & $86(25.9)$ \\
Obesity, $\mathrm{n}(\%)$ & $187(33.3)$ & $112(33.7)$ \\
Hypertension, $\mathrm{n}(\%)$ & $411(73.3)$ & $238(71.7)$ \\
Hyperlipidemia, n (\%) & $143(25.5)$ & $86(25.9)$ \\
Diabetes, $\mathrm{n}(\%)$ & $82(14.6)$ & $50(15.1)$ \\
Obstructive CAD, n $(\%)$ & $51(9.1)$ & $28(8.4)$ \\
Stroke/TIA, n $(\%)$ & $43(7.7)$ & $27(8.1)$ \\
Thyroid gland disease, $\mathrm{n}(\%)$ & $56(10.0)$ & $36(10.8)$ \\
eGFR $<60 \mathrm{ml} / \mathrm{min} / 1.73 \mathrm{~m}^{2}$ & $138(24.6)$ & $83(25.0)$ \\
Imaging parameters & Imaging parameters & Imaging parameters \\
LVEF $<50 \%, \mathrm{n}(\%)$ & $71(12.7)$ & $23(6.9)$ \\
iLAV $\left(\mathrm{ml} / \mathrm{m}^{2}\right)$ & $57.4 \pm 21.6$ & $54.4 \pm 19.3$ \\
LAAV $(\mathrm{ml})$ & $8.1 \pm 4.2$ & $7.6 \pm 3.2$ \\
LAA orifice area $\left(\mathrm{mm}^{2}\right)$ & $414.9 \pm 155.6$ & $387.6 \pm 140.5$ \\
LAA flow velocity $\left(\mathrm{cm}^{2} / \mathrm{sec}\right)$ & $34.1 \pm 13.0$ & $34.1 \pm 13.2$ \\
\hline
\end{tabular}

Abbreviations: $\mathrm{AAD}=$ anti-arrhythmic drug; $\mathrm{AF}=$ atrial fibrillation; $\mathrm{CAD}=$ coronary artery disease; eGFR = estimated glomerular filtration rate; iLAV = body surface area-indexed left atrial volume; LAA = left atrial appendage; LAAV = left atrial volume; $\mathrm{LVEF}=$ left ventricular ejection fraction; TIA = transient ischemic attack. 
Supplementary table 1.

\section{Medications}

\begin{tabular}{ll}
\hline NOAC, n (\%) & $312(55.6)$ \\
OAC, n (\%) & $237(42.2)$ \\
Syncumar, n (\%) & $74(13.2)$ \\
Warfarin, n (\%) & $121(21.6)$ \\
Dabigatran, n (\%) & $113(20.1)$ \\
Rivaroxaban, n (\%) & $100(17.8)$ \\
LMWH, n (\%) & $251(44.7)$ \\
Antiplatelet therapy, n (\%) & $13(2.3)$ \\
Apixaban, n (\%) & $24(4.3)$ \\
AAD therapy, n (\%) & $283(50.4)$ \\
Sotalol, n (\%) & $47(8.4)$ \\
Amiodarone, n (\%) & $139(24.8)$ \\
Propafenon, n (\%) & $97(17.3)$ \\
Procedural times & Procedural times \\
Procedure time (min) & $94.7 \pm 28.1$ \\
LA time (min) & $64.8 \pm 23.5$ \\
Fluoroscopy time (min) & $9.7 \pm 6.5$ \\
\hline
\end{tabular}

Abbreviations: $\mathrm{LA}=$ left atrium; $\mathrm{LMWH}=$ light molecular weight heparin; NOAC $=$ non-Vitamin $\mathrm{K}$ antagonist oral anticoagulation; $\mathrm{OAC}=$ oral anticoagulant.

Table 2. Clinical characteristics by AF type

\begin{tabular}{llll}
\hline & Paroxysmal AF (n=376) & Persistent AF (n=185) & $\mathrm{p}$ \\
\hline Age $>65$ years, n (\%) & $154(41.0)$ & $94(50.8)$ & $\mathbf{0 . 0 3 0}$ \\
Female, n (\%) & $136(36.2)$ & $60(32.4)$ & 0.398 \\
Obesity, n (\%) & $132(35.1)$ & $55(29.7)$ & 0.217 \\
Hypertension, n (\%) & $252(67.0)$ & $159(85.9)$ & $<\mathbf{0 . 0 0 1}$ \\
Hyperlipidemia, n (\%) & $86(22.9)$ & $57(30.8)$ & 0.050 \\
Diabetes, n (\%) & $49(13.0)$ & $33(17.8)$ & 0.162 \\
Obstructive CAD, n (\%) & $34(9.0)$ & $17(9.2)$ & 1.000 \\
Stroke/TIA, n (\%) & $32(8.5)$ & $11(5.9)$ & 0.316 \\
Thyroid gland disease, n (\%) & $46(12.2)$ & $10(5.4)$ & 0.011 \\
eGFR $<60$ ml/min/1.73 m ${ }^{2}$ & $100(26.6)$ & $38(20.5)$ & 0.144 \\
Pre-ablation AAD therapy, n (\%) & $196(52.1)$ & $87(47.0)$ & 0.281 \\
LVEF $<50 \%$, n (\%) & $25(6.6)$ & $46(24.9)$ & $<\mathbf{0 . 0 0 1}$ \\
\hline
\end{tabular}

Abbreviations: $\mathrm{AAD}=$ anti-arrhythmic drug; $\mathrm{AF}=$ atrial fibrillation; $\mathrm{CAD}=$ coronary artery disease; eGFR = estimated glomerular filtration rate; $\mathrm{LVEF}=$ left ventricular ejection fraction; TIA $=$ transient ischemic attack.

Table 3. Associates of AF recurrence in patients with paroxysmal AF

\begin{tabular}{lllll}
\hline Paroxysmal AF & Paroxysmal AF & Paroxysmal AF & Paroxysmal AF \\
\hline Unadjusted analysis & Unadjusted analysis & Unadjusted analysis & Adjusted analy \\
HR & $95 \%$ CI & p & HR
\end{tabular}




\begin{tabular}{|c|c|c|c|c|}
\hline & Paroxysmal AF & Paroxysmal AF & Paroxysmal AF & Paroxysmal AF \\
\hline Age $>65$ years, n $(\%)$ & 1.01 & $0.71-1.42$ & 0.967 & 1.02 \\
\hline Female, $\mathrm{n}(\%)$ & 1.43 & $1.01-2.02$ & 0.043 & 1.42 \\
\hline Obesity, n (\%) & 1.08 & $0.75-1.54$ & 0.685 & 1.09 \\
\hline Hypertension, n (\%) & 0.96 & $0.66-1.39$ & 0.816 & 0.91 \\
\hline Hyperlipidemia, n (\%) & 0.82 & $0.54-1.24$ & 0.338 & 0.86 \\
\hline Diabetes, n (\%) & 0.77 & $0.45-1.33$ & 0.353 & 0.79 \\
\hline Obstructive CAD, n (\%) & 1.08 & $0.62-1.89$ & 0.781 & 1.28 \\
\hline Stroke/TIA, n (\%) & 0.88 & $0.47-1.62$ & 0.673 & 0.96 \\
\hline Thyroid gland disease, $\mathrm{n}(\%)$ & 0.94 & $0.55-1.58$ & 0.806 & 0.85 \\
\hline $\mathrm{eGFR}<60 \mathrm{ml} / \mathrm{min} / 1.73 \mathrm{~m}^{2}$ & 1.38 & $0.93-2.06$ & 0.111 & 1.30 \\
\hline Pre-ablation AAD therapy, n (\%) & 0.96 & $0.68-1.36$ & 0.836 & 1.00 \\
\hline $\mathrm{LVEF}<50 \%, \mathrm{n}(\%)$ & 1.67 & $0.96-2.91$ & 0.069 & 1.42 \\
\hline $\mathrm{iLAV}\left(\mathrm{ml} / \mathrm{m}^{2}\right)$ & 1.01 & $0.99-1.02$ & 0.098 & 1.01 \\
\hline $\mathrm{LAAV}(\mathrm{ml})$ & 1.03 & $0.97-1.09$ & 0.325 & 1.00 \\
\hline LAA orifice area $\left(\mathrm{mm}^{2}\right)$, per $10 \mathrm{~mm}^{2}$ & 1.01 & $1.00-1.03$ & 0.034 & 1.00 \\
\hline LAA flow velocity $(\mathrm{cm} / \mathrm{sec})$ & 1.00 & $0.99-1.03$ & 0.352 & 1.01 \\
\hline
\end{tabular}

Abbreviations: $\mathrm{AAD}=$ anti-arrhythmic drug; $\mathrm{AF}=$ atrial fibrillation; $\mathrm{BMI}=$ body mass index; $\mathrm{CAD}=$ coronary artery disease; iLAV = body surface area-indexed left atrial volume; LAA = left atrial appendage; $\mathrm{LAAV}=$ left atrial volume; $\mathrm{LVEF}=$ left ventricular ejection fraction; TIA $=$ transient ischemic attack. 